\title{
The Constitutive Activation of Egr-1/C/EBPa Mediates the Development of Type 2 Diabetes Mellitus by Enhancing Hepatic Gluconeogenesis
}

Ning Shen, Shan Jiang, Jia-Ming Lu, Xiao Yu, Shan-Shan Lai, Jing-Zi Zhang, Jin-Long Zhang, Wei-Wei Tao, Xiu-Xing Wang, $\mathrm{Na} \mathrm{Xu}$, Bin Xue, and Chao-Jun Li

Accepted for publication

October 2, 2014.

Address correspondence to Bin Xue, Ph.D., or Chao-Jun Li, Ph.D., Model Animal Research Center and School of Medicine, Nanjing University, Nanjing 210093, China. E-mail: xuebin@nju.edu.cn or licj@nju. edu.cn.

\begin{abstract}
The sequential secretion of insulin and glucagon delicately maintains glucose homeostasis by inhibiting or enhancing hepatic gluconeogenesis during postprandial or fasting states, respectively. Increased glucagon/insulin ratio is believed to be a major cause of the hyperglycemia seen in type 2 diabetes. Herein, we reveal that the early growth response gene-1 (Egr-1) can be transiently activated by glucagon in hepatocytes, which mediates glucagon-regulated gluconeogenesis by increasing the expression of gluconeogenesis genes. Blockage of Egr-1 function in the liver of mice led to lower fasting blood glucose, better pyruvate tolerance, and higher hepatic glycogen content. The mechanism analysis demonstrated that Egr-1 can directly bind to the promoter of C/EBPa and regulate the expression of gluconeogenesis genes in the later phase of glucagon stimulation. The transient increase of Egr-1 by glucagon kept the glucose homeostasis after fasting for longer periods of time, whereas constitutive Egr-1 elevation found in the liver of $d b / d b$ mice and high serum glucagon level overactivated the $C / E B P a / g l u c o n e o g e n e s i s$ pathway and resulted in hyperglycemia. Blockage of Egr-1 activation in prediabetic $d b / d b$ mice was able to delay the progression of diabetes. Our results suggest that dysregulation of Egr-1/C/EBPa on glucagon stimulation may provide an alternative mechanistic explanation for type 2 diabetes. (Am J Pathol 2015, 185: 513-523; http://dx.doi.org/10.1016/j.ajpath.2014.10.016)
\end{abstract}

Glucose homeostasis is maintained by hormonal regulation of the balance between glucose production by the liver and glucose uptake by muscle and adipocytes. ${ }^{1}$ Insulin is responsible for glucose use after feeding and lowers the circulating glucose level, whereas glucagon is the major hormone that elevates the blood glucose level by regulating gluconeogenesis and glycogenolysis in the liver during the fasting condition. It has been widely accepted that the insulin resistance of peripheral tissues is a primary cause of type 2 diabetes. $^{2-4}$ Nevertheless, the dysregulation of the gluconeogenesis by glucagon has recently been recognized to contribute to the development of insulin resistance.

Diabetic patients normally exhibit enhanced hepatic gluconeogenesis and higher blood glucose levels during the fasting condition. 6,7 The hyperglycemia during fasting in type 2 diabetes patients is due to the elevated ratio of circulating glucagon/insulin, ${ }^{8}$ which is regarded as a crucial determinant of the circulating glucose level. Fasting hyperglucagonemia with impaired postprandial glucagon suppression due to defective insulin action contributes to hyperglycemia in type 2 diabetes patients. ${ }^{9-11}$ Furthermore, streptozocin-treated $\mathrm{Gcgr}^{-/-}$mice or mice lacking glucagon showed even better glucose disposal in glucose tolerance tests (GTTs), despite the absence of an increase in plasma insulin. ${ }^{12,13}$ Thus, the increased insulin level during type 2 diabetes is largely directed toward overcoming hepatic glucagon action. ${ }^{14}$

\footnotetext{
Supported by Chinese National Program on Key Basic Research Project (973 Program) grant 2012CB524900 (C.-J.L.), National Natural Science Foundation of China grant 31171306, Nature Science Foundation of Jiangsu Province grant BK2011568 (B.X.), and the Nanjing University program for outstanding doctoral (Ph.D.) candidates.

N.S. and S.J. contributed equally to this work.

Disclosures: None declared.
} 
We have reported that hyperinsulinemia could constitutively activate the function of early growth response gene-1 (Egr-1) and enhanced insulin resistance in adipocytes. ${ }^{15}$ The studies in conventional knockout mice reveal that obesity-induced Egr-1 promotes energy storage in adipocytes and that loss of Egr-1 protects against diet-induced insulin resistance and hyperlipidemia. ${ }^{16}$ Egr-1 is also involved in the inhibition of $5^{\prime}$-adenosine monophosphate-activated protein kinase agonist to hepatic gluconeogenic gene expression. ${ }^{17} \mathrm{Egr}-1$ is a member of the immediate early gene family identified by zinc finger domains, which recognize the highly conserved consensus GC-rich sequence $[\mathrm{GCG}(\mathrm{G} / \mathrm{T}) \mathrm{GGGCG}],{ }^{18,19}$ to enhance the expression of many target genes, such as TNF, PTEN, and GGPS1. ${ }^{20-22}$ In this manner, Egr-1 is involved in cell proliferation, apoptosis, tumorigenesis, and cell metabolism..$^{23,24}$ Egr-1 can be transiently activated through the mitogenactivated protein kinase pathway by a series of stimuli, such as cytokines and hormones, including insulin, glucagon-like peptide-1, and its potent agonist, exendin- $4 .^{25-27}$ When we inhibited the transcriptional activity of Egr-1 with its dominantnegative (DN) form in diabetic $d b / d b$ mice, the systemic insulin sensitivity was enhanced and the postprandial blood glucose level was significantly decreased. ${ }^{28}$ Meanwhile, we found that the fasting blood glucose level was further decreased, although there was no significant difference. Thus, we hypothesize that Egr-1 and its target genes may also be involved in the regulation of fasting blood glucose levels.

Herein, we examined whether and how hepatic Egr-1 and its target genes regulate glucagon-induced gluconeogenesis under fasting stress. Although there are no direct binding sites of Egr-1 on the promoters of the gluconeogenic genes, we reveal that Egr-1 can directly bind to the promoter of $C / E B P a$, a gluconeogenic transcription factor, to indirectly activate the expression of gluconeogenic genes. More important, early intervention against hepatic Egr-1 in prediabetic $d b / d b$ mice could ameliorate the hyperglycemia and insulin resistance by inhibiting hepatic gluconeogenesis.

\section{Materials and Methods}

\section{Materials}

The protease inhibitor cocktail was from Roche Diagnostics Ltd (Mannheim, Germany); anti-Egr-1 (sc-189), anti-actin (sc-47778), and alkaline phosphatase-conjugated secondary antibody (sc-2007/2008) were from Santa Cruz Biotechnology (Santa Cruz, CA); horseradish peroxidase-tagged secondary antibody (P0160/0161) was from Dako Cytomation (Glostrup, Denmark); the glucose assay kit (K686-100) and glycogen assay kit (K648-100) were from Biovision (Mountain View, CA); insulin (40 IU/mL) was from Novolin (Bagsaerd, Denmark); and glucagon was from Merck Millipore (Billerica, MA). Lactate and pyruvate were from Sigma (St. Louis, MO). The glucometer and blood glucose test strips were from Roche Diagnostics Ltd. The Invivofectamine 2.0 reagent was from Invitrogen (Carlsbad, CA). The siRNAs purchased from Invitrogen were designed to target the following cDNA sequences: Egr-1, 5'-TCTCCCAGGACAATTGAAATTTGCT-3'; C/EBPa, 5'-CGACGAGTTCCTGGCCGAC-3'; and Scrambled, 5'-TTCTCCGAACGTGTCACGT-3'. Wild-type (WT) - and DN-Egr-1 cDNAs were a gift from Prof. Jay M. Baraban (Johns Hopkins University, Baltimore, MD). WT- and DN-Egr-1 adenoviruses were constructed in our laboratory with the AdEasy System (Agilent Technology, Palo Alto, CA), according to the manufacturer's protocol. The production of DN-Egr-1 adenovirus could compete with the WT-Egr-1 to bind to the promoter of target genes but had no transcriptional activity.

\section{Mice}

Male $d b / d b$ mice (C57BL/KsJ.Cg-m+/+Lepr db) and control littermates were purchased from the Model Animal Research Center of Nanjing University (Nanjing, China). All of the mice were maintained on a 12-hour light-dark cycle. All protocols for animal use were reviewed and approved by the Animal Care Committee of Nanjing University in accordance with Institutional Animal Care and Use Committee guidelines. WT and $d b / d b$ mice ( 3 or 6 weeks old) were i.v. injected with $200 \mu \mathrm{L}$ of Egr-1 siRNA or control siRNA $(70 \mu \mathrm{g})$ through Invivofectamine 2.0 reagent (Invitrogen). To ensure that the siRNA was concentrated in the liver, the siRNA was labeled by cholesterol. ${ }^{29}$ The use of Invivofectamine 2.0 as the transfection reagent in vivo could further increase the concentration of siRNA in the liver without affecting other tissues. Mice were injected once a week $(8 \mathrm{mg} / \mathrm{kg})$ until being sacrificed at the indicated time point. Recombinant adenovirus vector bearing $\mathrm{DN}-\mathrm{Egr}-1$ was generated as described above. C57BL/6 mice (6 weeks old) were i.v. injected with $200 \mu \mathrm{L}$ of the recombinant adenovirus $\left(2 \times 10^{9}\right.$ plaque-forming units $)$. Mice were injected once a week until sacrificed at the indicated time point. The mice were maintained on a standard diet $(65 \%$ carbohydrate, $4 \%$ fat, $24 \%$ protein, and $7 \%$ crude fiber). Blood was obtained from the vena cava, and the plasma was stored at $-80^{\circ} \mathrm{C}$ until analysis. The body weight of the mice was also measured when the mice were sacrificed.

\section{Primary Hepatocyte Culture}

Mice were anesthetized ( $300 \mathrm{mg} / \mathrm{kg}$ chloral hydrate) by i.p. injection. Hepatocytes were isolated from the livers as described previously. ${ }^{30}$ Briefly, the liver was perfused with perfusion buffer ( $\mathrm{pH} 7.5$ ) containing $137 \mathrm{mmol} / \mathrm{L} \mathrm{NaCl}, 5.4$ $\mathrm{mmol} / \mathrm{L} \mathrm{KCl}, 0.5 \mathrm{mmol} / \mathrm{L} \mathrm{NaH}_{2} \mathrm{PO}_{4}, 0.42 \mathrm{mmol} / \mathrm{L} \mathrm{Na}_{2} \mathrm{HPO}_{4}$, $10 \mathrm{mmol} / \mathrm{L}$ HEPES, $0.5 \mathrm{mmol} / \mathrm{L}$ EGTA, $4.2 \mathrm{mmol} / \mathrm{L}$ $\mathrm{NaHCO}_{3}$, and $5 \mathrm{mmol} / \mathrm{L}$ glucose, and with collagenase buffer (pH 7.5) containing $137 \mathrm{mmol} / \mathrm{L} \mathrm{NaCl}, 5.4 \mathrm{mmol} / \mathrm{L} \mathrm{KCl}, 5$ $\mathrm{mmol} / \mathrm{L} \quad \mathrm{CaCl}_{2}, \quad 0.5 \mathrm{mmol} / \mathrm{L} \quad \mathrm{NaH}_{2} \mathrm{PO}_{4}, 0.42 \mathrm{mmol} / \mathrm{L}$ $\mathrm{Na}_{2} \mathrm{HPO}_{4}, 10 \mathrm{mmol} / \mathrm{L}$ HEPES, $0.15 \mathrm{~g} / \mathrm{L}$ collagenase IV (Sigma), and $4.2 \mathrm{mmol} / \mathrm{L} \mathrm{NaHCO}_{3}$ through a needle aligned along the inferior vena cava. The collagenase-perfused liver 
was then dissected, suspended in phosphate-buffered saline (PBS; $30 \mathrm{~mL}$ ), and filtered through cheesecloth and a $100-\mu \mathrm{m}$ nylon membrane to remove connective tissue debris and cell clumps. Hepatocytes were subjected to centrifugation $(50 \times g$ for 2 minutes at $4{ }^{\circ} \mathrm{C}$ ) and resuspended in Dulbecco's modified Eagle's medium (DMEM) supplemented with $10 \%$ fetal bovine serum and penicillin $(100 \mathrm{IU} / \mathrm{mL}) /$ streptomycin $(100$ $\mathrm{ng} / \mathrm{mL}$ ). This protocol was repeated three times. Cell viability, measured by Trypan blue exclusion, was $>90 \%$. Then, the primary hepatocytes were cultured in DMEM medium $(4.5 \mathrm{~g} / \mathrm{L}$ glucose) with $10 \%$ fetal bovine serum and penicillin (100 IU/ $\mathrm{mL}) /$ streptomycin $(100 \mathrm{ng} / \mathrm{mL})$ at $37^{\circ} \mathrm{C}$ in a water-saturated atmosphere with 5\% carbon dioxide. For adenovirus infection, cells were exposed to adenovirus at a multiplicity of infection of 200 in media overnight. For knockdown studies, cells were transfected with control or C/EBPa siRNA (in lowserum Opti-MEM using Lipo2000 reagent from Invitrogen) according to the manufacturer's recommendations.

\section{Glucagon Administration in Vitro}

The glucagon purchased from Merck Millipore (Billerica, MA) was dissolved at $100 \mathrm{mmol} / \mathrm{L}$ as the manufacturer indicated. In the time-dependent experiment, the final concentration in the medium was $160 \mathrm{nmol} / \mathrm{L}$. Before glucagon was administrated, the cells were starved in serum-free DMEM (supplemented with $100 \mathrm{IU} / \mathrm{mL}$ penicillin and 100 $\mathrm{ng} / \mathrm{mL}$ streptomycin) overnight.

\section{Cell Lysates and Immunoblotting}

Cells were washed in ice-cold PBS and harvested with radioimmunoprecipitation assay buffer containing sodium vanadate $(1 \mathrm{mmol} / \mathrm{L})$ and protease inhibitors (Roche Diagnostics Ltd). Cell or tissue protein extracts $(250 \mathrm{mg}$ total protein) were boiled in loading buffer. Protein $(50 \mu \mathrm{g}$ total protein per well) was separated by $10 \%$ SDS-PAGE and then transferred onto polyvinylidene difluoride membranes (BioRad, Richmond, CA). The membranes were incubated overnight with the indicated primary antibodies. Bound antibodies were then visualized using horseradish peroxidase-tagged secondary antibodies and film exposure. All of the antibodies were diluted in PBS with $0.1 \%$ Tween 20 . The dilution used was as followed: Egr-1 (1:500), actin (1:1000), and the secondary antibodies (1:5000).

\section{RNA Extraction and Gene Expression}

RNA was extracted from cells and tissues using TRIzol reagent (Invitrogen), according to the manufacturer's protocol. cDNA was synthesized from total RNA with PrimerScript RT Master Mix Perfect Real Time (TaKaRa, Tokyo, Japan). cDNAs were amplified with SYBR Green Master Mix (TaKaRa) in an ABI 7300 Prism real-time PCR instrument (ABI, Foster City, CA). All data were normalized to the expression of actin. Primer sequences for real-time PCR are as followed: Egr-1,
5'-TCGGCTCCTTTCCTCACTCA-3' (sense) and 5'-CTCATAGGGTTGTTCGCTCGG-3' (antisense); C/EBPa, 5'-CAAGAACAGCAACGAGTACCG-3' (sense) and 5'-GTCACTGGTCAACTCCAGCAC-3' (antisense); glucose-6phosphatase catalytic subunit (G6Pase), 5'-CGACTCGCTATCTCCAAGTGA-3' (sense) and 5'-GTTGAACCAGTCTCCGACCA-3' (antisense); phosphoenolpyruvate carboxykinase (PEPCK), 5'-CTGCATAACGGTCTGGACTTC-3' (sense) and 5'-CAGCAACTGCCCGTACTCC-3' (antisense); fructose-1,6-bisphosphatase 1 (FBP1), 5'-CACCGCGATCAAAGCCATCT-3' (sense) and 5'-AGGTAGCGTAGGACGACTTCA-3' (antisense); hepatocytes nuclear factors $4 \mathrm{a}$ (HNF-4a), 5'-CACGCGGAGGTCAAGCTAC-3' (sense) and $5^{\prime}$-CCCAGAGATGGGAGAGGTGAT- $3^{\prime}$ (antisense); and actin, 5'-CACCCGCGAGCACAGCTTCT-3' (sense) and 5'-TTGCACATGCCGGAGCCGTT-3' (antisense).

\section{Chromosome Immunoprecipitation}

Cells were fixed with $37 \%$ formaldehyde and lysed with $1 \%$ SDS. The lysates were subjected to ultrasonication (10 seconds, $22 \%$ power, $5 \times$ ) and centrifuged at $12,000 \times g$ for 10 minutes. The supernatant was immunoprecipitated with anti-Egr-1 antibody or control IgG, overnight. The supernatant that was not subjected to immunoprecipitation was the control DNA ( $2 \%$ input). The chromatin immunoprecipitation DNA and control DNA (2\% input) were analyzed using PCR and real-time PCR. Real-time PCR was performed using SYBR Green Master Mix (TaKaRa) in the ABI 7300 Prism real-time PCR instrument. The PCR ran for 22 cycles to ensure amplification in the linear range, and the primers used were as follows: 5'-CAACGTCTGCCTGGTAAG-3' (sense) and 5'-ACGGGCTCTTCAGAGTAGTAG-3' (antisense).

\section{Metabolite Determinations}

We cultured primary hepatocytes in DMEM supplemented with $10 \%$ fetal bovine serum and measured glucose production in glucose and phenol red-free DMEM (pH 7.4) supplemented with sodium lactate and pyruvate using a colorimetric assay (Biovision). The glycogen content of liver tissue was measured with a glycogen assay kit (Biovision), according to the manufacturer's recommendations.

\section{PTTs and GTTs}

The mice were fasted overnight. For the pyruvate tolerance test (PTT) or GTT, sodium pyruvate (2 g/kg; Sigma) or glucose ( $2 \mathrm{~g} / \mathrm{kg}$; Sigma), respectively, was administered by i.p. injection. The blood glucose level was measured every 30 minutes after injection.

\section{ITTs}

For the insulin tolerance test (ITT), insulin ( $0.8 \mathrm{U} / \mathrm{kg}$; Novolin) was administered to the fed mice by i.p. injection. The blood glucose level was measured every 30 minutes after injection. 
A

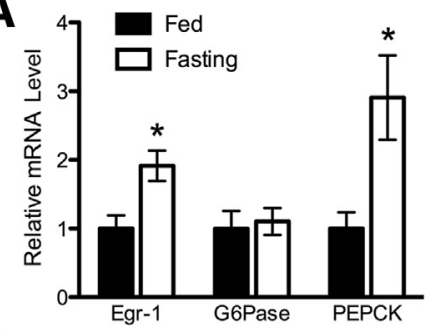

C
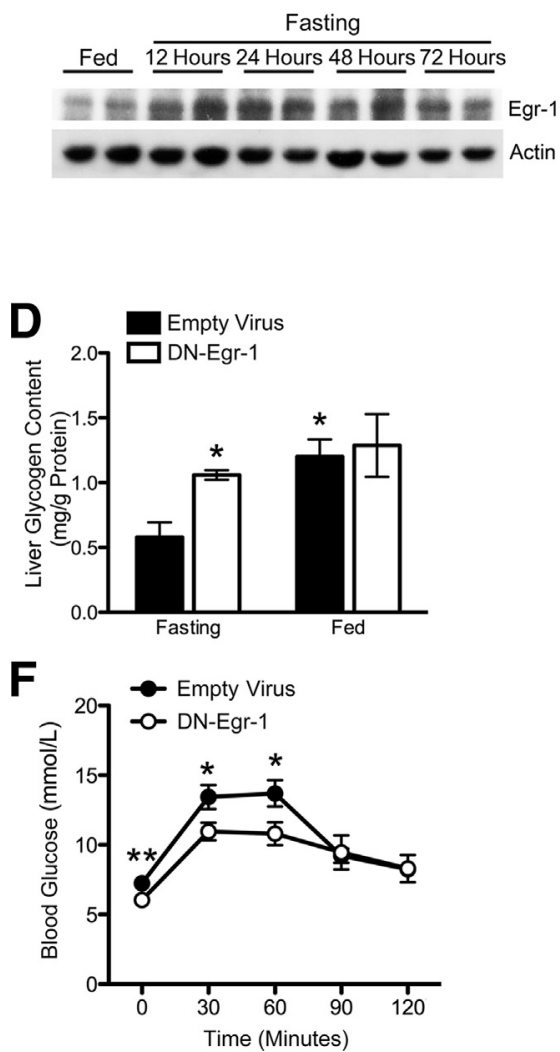

B
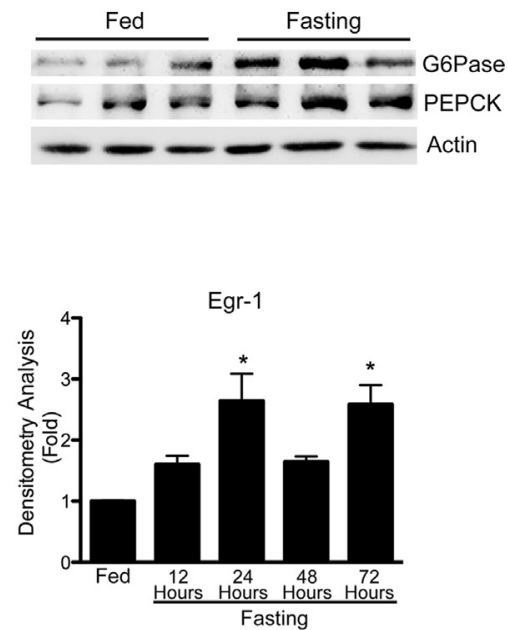

$\mathbf{E}$

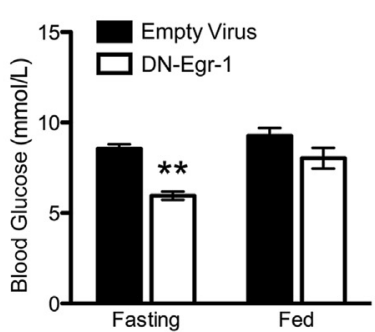

G

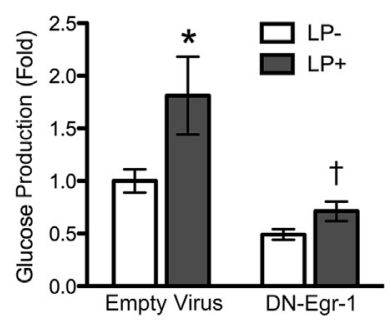

Figure 1 Early growth response growth gene-1 (Egr-1) is responsible for hepatic glucose production during the fasting state. A: $(\mathrm{C} 57 \mathrm{BL} / 6)$ mice were fasted overnight before sacrifice. The total mRNA of the liver tissue was extracted and subjected to real-time quantitative $P C R$ with the primers for Egr-1, glucose-6-phosphatase catalytic subunit (G6Pase), and phosphoenolpyruvate carboxykinase (PEPCK). B: C57BL/6 mice were fasted overnight before sacrifice. The protein of the liver tissue was extracted and subjected to Western blot analysis against G6Pase and PEPCK. C: C57BL/6 mice were fasted for the indicated time periods before sacrifice. The protein of the liver tissue was subjected to Western blot analysis to determine the Egr-1 level. The densitometry analysis of Egr-1 protein level. D: The glycogen content in the liver of the indicated mice was determined with a glycogen kit from BioVision. E: The adenovirus carrying dominant-negative (DN)-Egr-1 or the empty adenovirus $\left(2 \times 10^{9}\right.$ plaque-forming units) was administered to $\mathrm{C} 57 \mathrm{BL} / 6$ mice through tail vein injections. Four days later, the fasting blood glucose level was measured after being fasted for 16 hours. F: The PTT was performed in the indicated mice as described above. Each mouse was i.p. injected with $2 \mathrm{~g} / \mathrm{kg}$ pyruvate. G: Primary hepatocyte cultures were infected with DN Egr-1 adenovirus for 48 hours. Glucose production was detected after administration of lactate and pyruvate (LP). Data are presented as means \pm SEM. A one-way analysis of variance was used for the statistics of densitometry analysis in $\mathbf{C}$; otherwise, $t$-test was used for the two-group comparisons. ${ }^{*} P<0.05$ versus fed (A and $\mathbf{C}) ;{ }^{*} P<0.05,{ }^{* *} P<0.01$ versus empty virus in fasting (D and $\mathbf{E}) ;{ }^{*} P<0.05,{ }^{*} P<0.01$ versus empty virus $(\mathbf{F}) ;{ }^{*} P<0.05$ versus empty virus without $\mathrm{LP}$ and ${ }^{\dagger} P<0.05$ versus empty virus with LP (G).

\section{Statistical Analysis}

Data were shown as means \pm SEM. The $t$-test for unequal variances was introduced to determine the statistical significance of differences between two groups. A one-way analysis of variance, followed by a post hoc test, was used for multiple group comparisons. Differences with $P<0.05$ were considered significant. Prism 5 software (GraphPad Software, Inc., La Jolla, CA) was used to perform statistical analysis. The number of mice used in this study was at least five per group. Each experiment was performed at least three times.

\section{Results}

Fasting Induces Egr-1 Elevation, Which Regulates Fasting Glucose Level by Mediating Hepatic Glucose Production

Previously, we showed that the fasting blood glucose level could be decreased by injecting diabetic $d b / d b$ mice with adenovirus carrying DN Egr- $1 .{ }^{28}$ When C57BL/6 mice were fasted overnight, we found that the expression level of Egr-1 was significantly increased in the liver (Figure 1A). This elevation of Egr-1 expression lasted for up to 72 hours during fasting (Figure 1C). Meanwhile, the gluconeogenic gene, such as PEPCK, was increased in both transcriptional and translational levels under fasting condition, whereas G6Pase was elevated just in translational level under fasting condition (Figure 1, A and B). This suggested that the glucose anabolic metabolism had been enhanced. Inhibition of Egr-1's transcriptional activity by tail vein injections with the adenovirus overexpressing DN Egr-1 led to inhibition of glycogenolysis (Figure 1D) and decreased the plasma glucose level (Figure 1E) during the fasting state, but not the fed state. The PTT showed that hepatic gluconeogenesis was impaired by DN-Egr-1 overexpression (Figure 1F). The in vitro measurement of glucose production in primary hepatocytes also showed that the loss of function of Egr-1 decreased hepatic glucose release in the context of pyruvate and lactate (Figure 1G). These results suggested that 
A
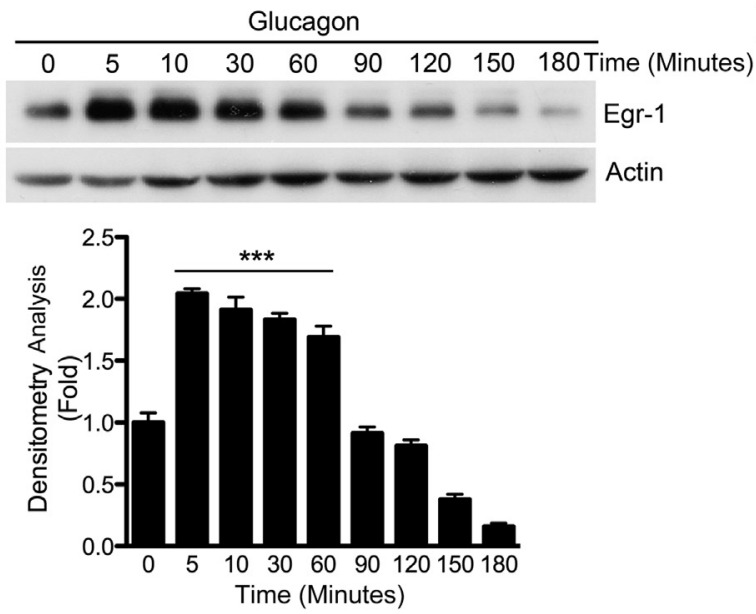

C

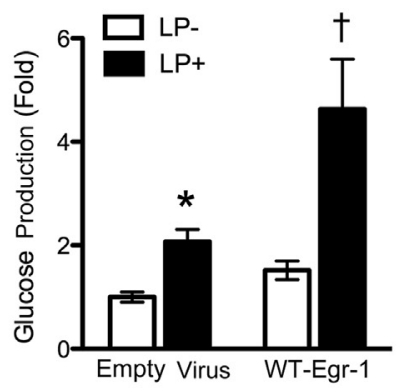

B
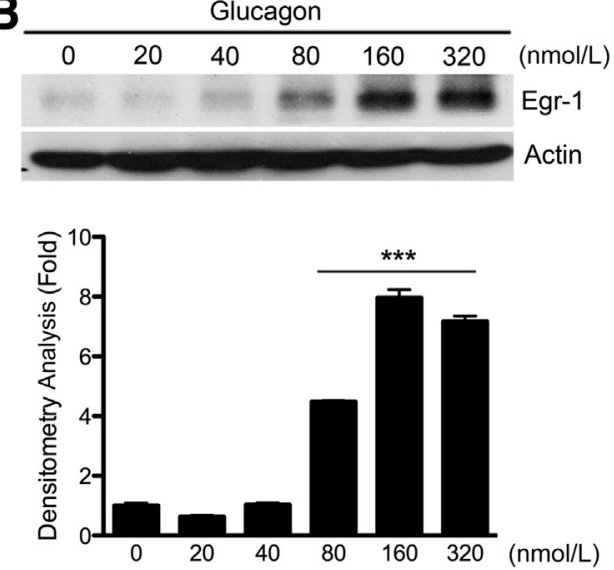

D

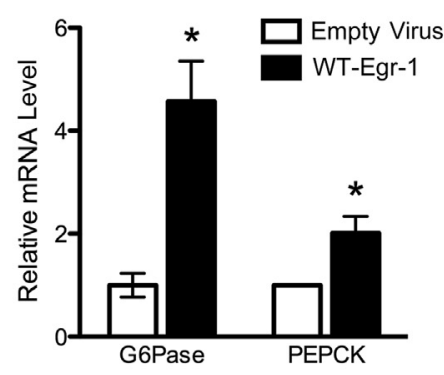

E

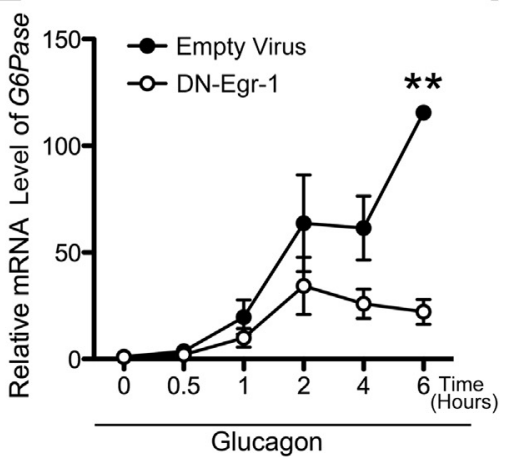

G

$F$

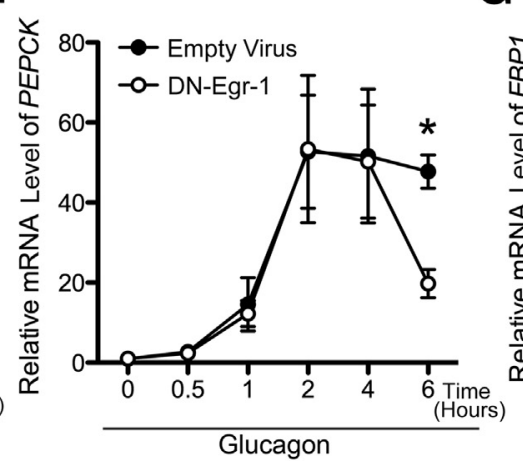

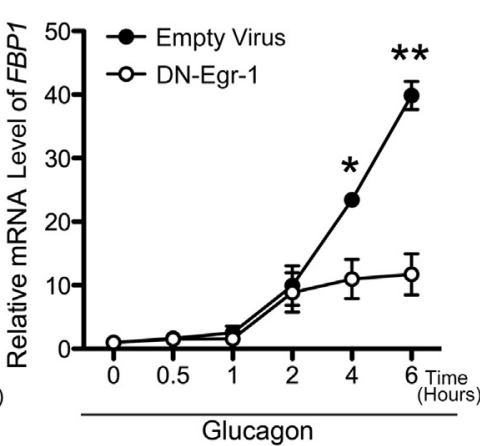

Figure 2 Early growth response growth gene-1 (Egr-1) is responsible for expression of hepatic gluconeogenic genes on glucagon stimulation. A: Primary cultured hepatocytes were treated with or without $160 \mathrm{nmol} / \mathrm{L}$ glucagon for the indicated time points. The cells were lysed and subjected to Western blot analysis to detect the level of Egr-1; densitometry analysis is below. B: Primary cultured hepatocytes were treated with or without $160 \mathrm{nmol} / \mathrm{L}$ glucagon at the indicated concentrations. The cells were lysed and subjected to Western blot analysis to detect the level of Egr-1; densitometry analysis is below. C: Primary hepatocytes were infected with empty or wild-type (WT)-Egr-1 adenovirus. Forty-eight hours later, glucose production was measured after administration of lactate and pyruvate (LP). D: Primary hepatocytes were infected with empty or WT-Egr-1 adenovirus. Total mRNA was extracted and subjected to real-time quantitative PCR (qPCR) with primers for glucose-6-phosphatase catalytic subunit (G6Pase) and phosphoenolpyruvate carboxykinase (PEPCK). E-G: The primary hepatocytes were infected with empty or dominant-negative (DN)-Egr-1 adenovirus 48 hours ahead of $160 \mathrm{nmol} / \mathrm{L}$ glucagon treatment for the indicated time points. The total mRNA was extracted and subjected to qPCR with the primers for G6Pase (E), PEPCK (F), and fructose-1,6-bisphosphatase 1 (FBP1) (G). Data are presented as means \pm SEM. A one-way analysis of variance was used for the statistics of densitometry analysis in $\mathbf{A}$ and $\mathbf{B}$; otherwise, $t$-test was used for the two-group comparisons. ${ }^{\star} P<0.001$ (A and $\left.\mathbf{B}\right) ;{ }^{*} P<0.05$ versus empty virus without $L P,{ }^{\dagger} P<0.05$ versus empty virus with $\mathrm{LP}(\mathbf{C}) ;{ }^{*} P<0.05$ versus empty virus $(\mathbf{D}) ;{ }^{*} P<0.05,{ }^{*} P P<0.01$ versus empty virus at the same time points (E-G).

Egr-1 could regulate the fasting glucose level by mediating hepatic gluconeogenesis.

\section{Glucagon Stimulates Egr-1 Activation, Which Is Responsible for Hepatic Gluconeogenic Gene Expression}

Glucagon was increased during fasting and is the major hormone responsible for hepatic gluconeogenesis. We found that glucagon stimulation could increase Egr-1 levels temporally and in a dose-dependent manner (Figure 2, A and B). The glucagon concentration of $160 \mathrm{nmol} / \mathrm{L}$ in the medium was chosen in the following experiments because Egr-1 was well responded without any cellular toxicity. The elevation of Egr-1 expression enhances glucose production in primary hepatocytes (Figure 2C) by enhancing the expression of gluconeogenic genes, such as G6Pase 
A

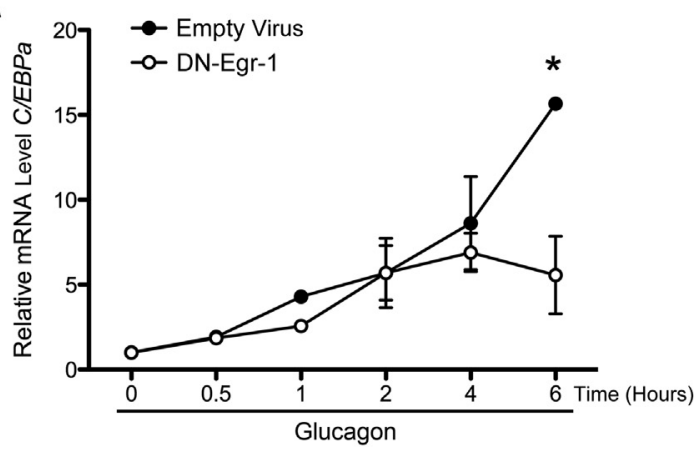

C
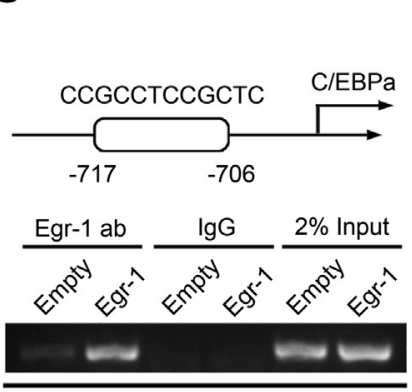

C/EBPa Promoter

G

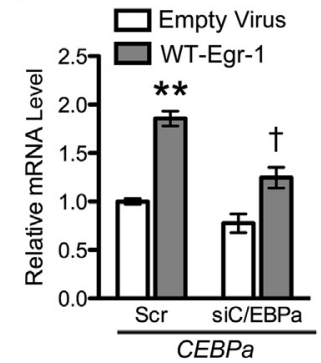

D

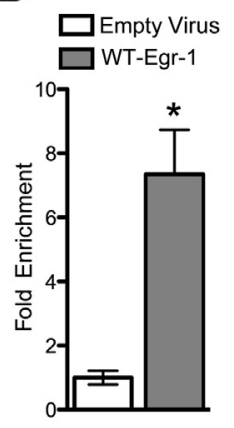

H

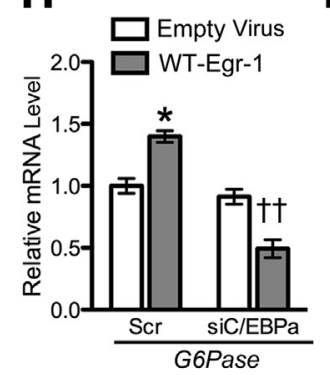

B

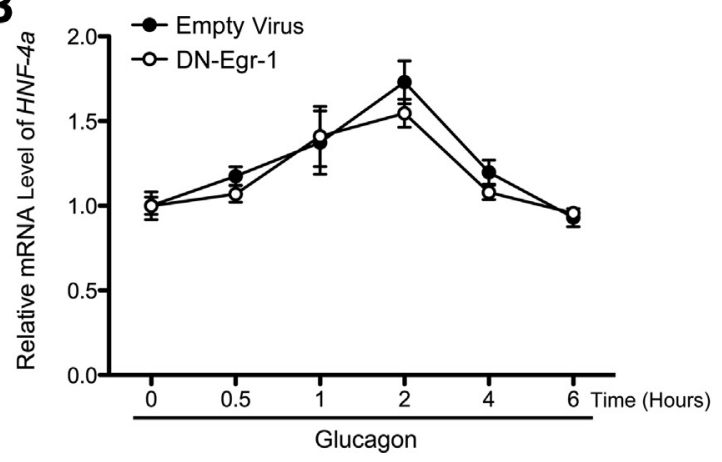

E

$\mathbf{F}$
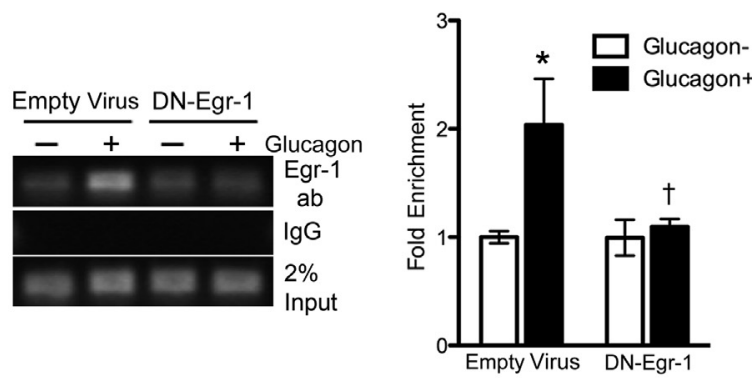

I

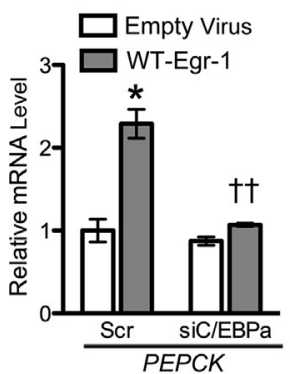

J

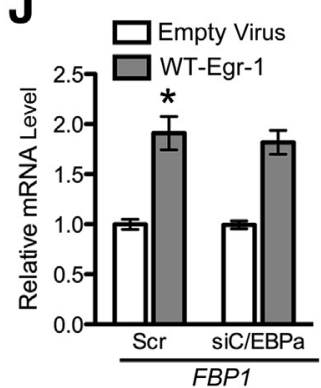

Figure 3 Early growth response growth gene-1 (Egr-1) can directly bind to the promoter of CCAAT/enhancer binding protein a (C/EBPa) and enhance its expression. A and B: Primary hepatocytes were infected with empty or dominant-negative (DN)-Egr-1 adenovirus 48 hours ahead of the glucagon treatment for the indicated time points. Total mRNA was extracted and subjected to real-time quantitative PCR (qPCR) with primers for C/EBPa (A) and hepatocytes nuclear factors 4a (HNF-4a) (B). C: Primary hepatocytes were infected with empty or wild-type (WT)-Egr-1 adenovirus for 48 hours. Chromatin immunoprecipitation (ChIP) was performed as described in Materials and Methods. D: ChIP DNA was subjected to qPCR to quantify the amount of Egr-1 binding to the promoter of C/EBPa. E: Primary hepatocytes were infected with empty or DN-Egr-1 adenovirus for 48 hours. After treatment with glucagon, ChIP was performed as described in Materials and Methods. F: ChIP DNA was subjected to GPCR to quantify the amount of Egr-1 binding to the promoter of C/EBPa. G-J: Primary hepatocytes were infected with empty or WT-Egr-1 adenovirus and transfected with C/EBPa siRNA or control at the same time for 48 hours. Then, RNA was extracted and subjected to qPCR. The expression levels of C/EBPa (G), glucose-6-phosphatase catalytic subunit (G6Pase; $\mathbf{H}$ ), phosphoenolpyruvate carboxykinase (PEPCK) (I), and fructose-1,6-bisphosphatase 1 (FBP1) (J) were detected. Data are presented as means \pm SEM. A $t$-test was used for the two group comparisons. ${ }^{*} P<0.05$ versus empty virus at the same time points (A); ${ }^{*} P<0.05$ versus empty virus (D); ${ }^{*} P<0.05$ versus empty virus without glucagon, ${ }^{\dagger} P<0.05$ versus empty virus with glucagon (F); ${ }^{*} P<0.05,{ }^{*} P<0.01$ versus empty virus in scramble (Scr) group, ${ }^{\dagger} P<0.05,{ }^{\dagger \dagger} P<0.01$ versus empty virus in siC/EBPa group (G-J).

(G6pc) and PEPCK (Pck2) (Figure 2D). Glucagon could also induce the expression of gluconeogenic genes, such as G6Pase, PEPCK, and FBP1 ( $F b p l)$, that are dependent on Egr-1, because loss of function of Egr-1 (through the expression of DN-Egr-1) largely reduced the expression of these genes (Figure 2, E-G). These observations suggested that Egr-1 could regulate glucagon function by mediating hepatic gluconeogenesis. We noticed that DN-Egr-1 could not inhibit gluconeogenic gene expression until 4 hours after glucagon stimulation, which suggested that the increased expression of gluconeogenic genes at the early stage of glucagon stimulation might not depend on Egr-1.

\section{Egr-1 Indirectly Enhances the Expression of Gluconeogenic Genes by Activating C/EBPa Transcription}

Although expression of gluconeogenic genes is dependent on Egr-1, we could not find any direct binding site for Egr-1 in the promoter of the G6Pase or PEPCK. Nevertheless, we 

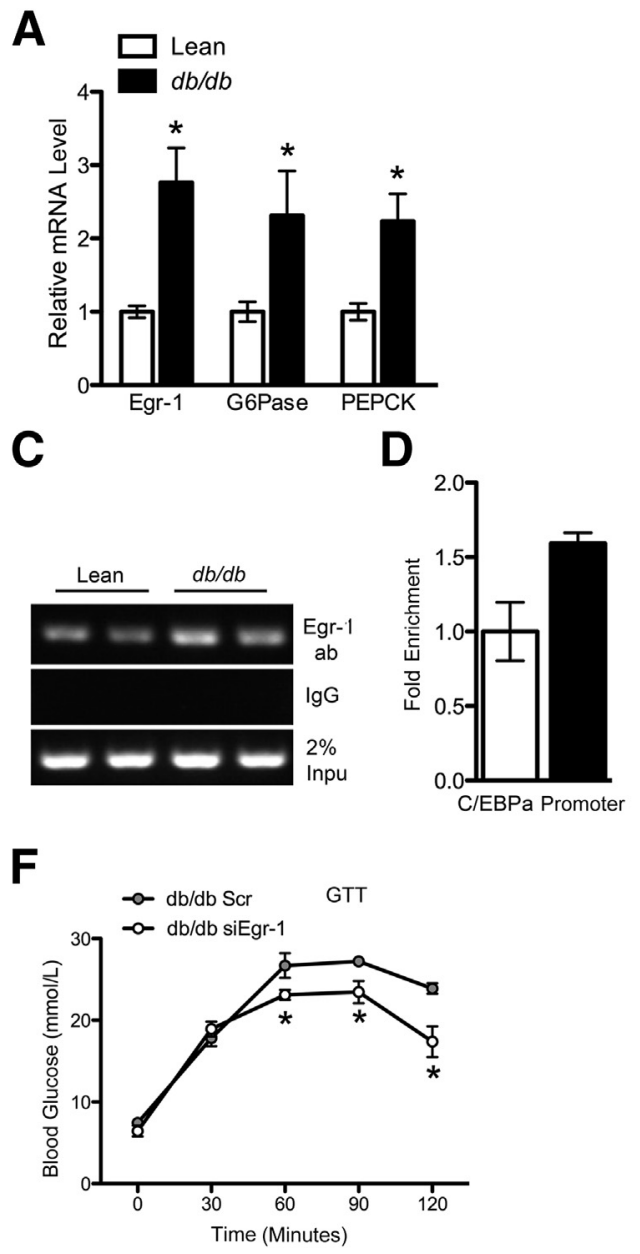

found that the gluconeogenic transcription factor, such as $\mathrm{C} / \mathrm{EBPa}$, was increased by glucagon treatment but downregulated by overexpression of DN-Egr-1 (Figure 3A), whereas another gluconeogenic transcription factor, HNF-4a, was not regulated by Egr-1 (Figure 3B). Peroxisome proliferator-activated receptor gamma, coactivator 1 alpha (PGC-1a) was also found to be regulated by Egr-1 (data not shown). Notably, there is a predicted binding site for Egr-1 in the promoter of $\mathrm{C} / \mathrm{EBPa}$, located at nucleotide positions 706 to 717 , but no predicted Egr-1 binding site of PGC-1a (Ppargcla) promoter was found. We overexpressed Egr-1 in primary hepatocytes and performed the chromatin immunoprecipitation assay with an anti-Egr-1 antibody. The chromatin immunoprecipitation DNA and the control DNA were subjected to PCR. The result suggested that Egr1 could directly bind to the predicted site on the promoter of C/EBPa (Cebpa) (Figure 3C). We further quantified the result with real-time quantitative PCR (Figure 3D). This binding of Egr-1 to the promoter of C/EBPa could also be enhanced by glucagon treatment, and this effect could be diminished by DN-Egr-1 overexpression (Figure 3, E and F). Further analysis suggested that Egr-1 promotion of gluconeogenic gene expression is dependent on $\mathrm{C} / \mathrm{EBPa}$ transcription; because Egr-1 boosted expression of G6Pase, PEPCK could be inhibited by $\mathrm{C} / \mathrm{EBPa}$ knockdown
(Figure 3, G-I). However, knockdown of C/EBPa in the Egr-1-overexpressed hepatocytes did not reduce the expression level of FBP1 (Figure 3J). This suggested that FBP1 might be regulated by Egr-1 in a C/EBPa-independent manner. Taken together, our results indicate that Egr-1 is a critical regulator of glucagon-stimulated gluconeogenesis.

\section{The Egr-1/C/EBPa/Gluconeogenesis Pathway Is Constitutively Activated in a Diabetic Mouse Model}

The glucagon/insulin ratio is increased in type 2 diabetes patients. Thus, we hypothesize that the development of type 2 diabetes might be promoted by constitutively activated Egr-1/ $\mathrm{C} / \mathrm{EBPa} /$ gluconeogenesis. The $d b / d b$ mouse is extensively used to study the mechanism of type 2 diabetes. Deficient leptin receptor activity causes $d b / d b$ mice to become obese and resistant to insulin stimulation at approximately 6 to 8 weeks of age. ${ }^{31}$ We found that Egr-1 was constitutively elevated in the 8-week-old diabetic $d b / d b$ mice (Figure 4, A and B). Also, the expression levels of the gluconeogenic genes, G6Pase and PEPCK, were significantly higher in $d b / d b$ mice than in control mice (Figure 4A). Increased Egr-1 was constitutively bound to the promoter of C/EBPa in the liver of $d b / d b$ mice (Figure 4, C and D). This binding was responsible for the expression of gluconeogenic genes, such as PEPCK 

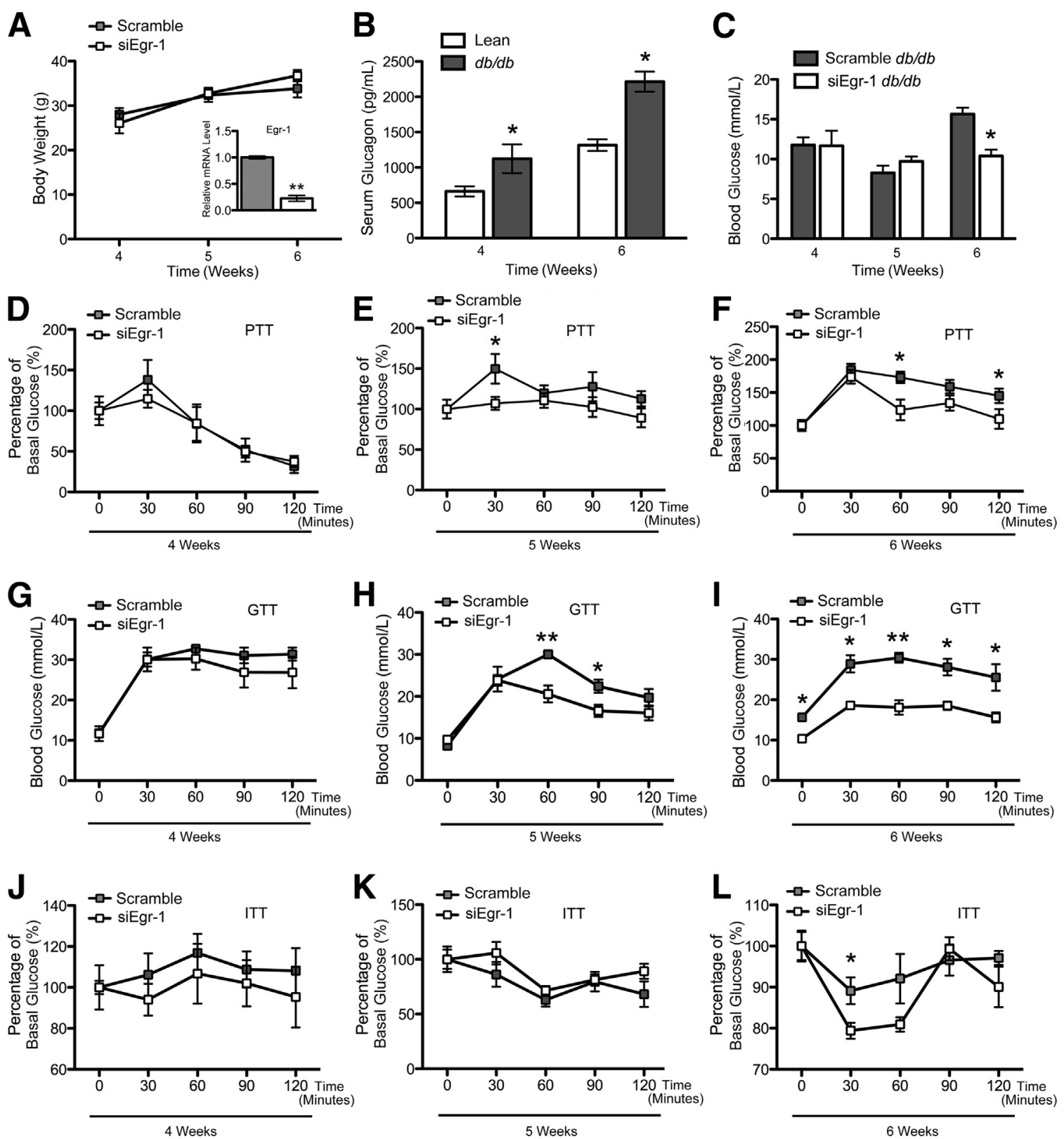

Figure 5 Early intervention through inhibition of hepatic early growth response growth gene-1 (Egr-1) expression alleviates the insulin resistance of $d b / d b$ mice. Three-week-old $d b / d b$ mice were injected with Egr-1 siRNA i.v. through weekly tail vein injections. During the following 3 weeks, the metabolic parameters of the mice were collected once a week. A: Body weight was measured, and the knockdown efficiency for hepatic Egr-1 at 6 weeks of age was detected. B: Serum glucagon was measured at 4 and 6 weeks of age. C: Fasting blood glucose levels were determined at 4, 5, and 6 weeks of age. D-F: Pyruvate tolerance tests (PTTs) were performed in mice that were 4 weeks old (D), 5 weeks old (E), and 6 weeks old (F). G-I: Glucose tolerance tests (GTTs) of 4-week-old (G), 5-week-old (H), and 6-week-old (I) mice were performed. J-L: Insulin tolerance tests (ITTs) were performed in mice that were 4 weeks old (J), 5 weeks old $(\mathbf{K})$, and 6 weeks old $(\mathbf{L})$. Data are presented as means \pm SEM. A $t$-test was used for the two group comparisons. ${ }^{*} P<0.05,{ }^{*} P<0.01$ versus scramble (A, C, E, F, H, I, and $\mathbf{L}) ;{ }^{*} P<0.05$ versus lean $(\mathbf{B})$.

(Figure 4E). To investigate the role of the constitutively activated Egr-1/C/EBPa pathway in insulin resistance, we knocked down Egr-1 with siRNA in the liver of 6-week-old diabetic $d b / d b$ mice. Unfortunately, GTTs and ITTs in 8 -week-old mice revealed only slightly improved glucose tolerance (Figure 4F) and enhanced insulin sensitivity (Figure 4G). Nevertheless, the above results suggested that the Egr-1/C/EBPa/gluconeogenesis pathway was constitutively activated in diabetic mice, although knockdown of hepatic
Egr-1 in well-developed diabetic $d b / d b$ mice had no positive effect on insulin resistance symptoms.

Early Intervention in Hepatic Egr-1 Expression Alleviates the Development of Type 2 Diabetes in $d b / d b$ Mice

Because knockdown of hepatic Egr-1 cannot rescue the insulin resistance symptoms in diabetic mice, we examined whether blocking the constitutively activated Egr-1/C/EBPa 
pathway could prevent the development of type 2 diabetes in $d b / d b$ mice. We began Egr-1 siRNA administration during the third week of life in $d b / d b$ mice, while they still lack any symptoms of type 2 diabetes, by injecting Egr-1 siRNA into the tail vein once a week until the mice were 6 weeks old. The metabolic parameters were measured by PTT, GTT, and ITT at 4, 5, and 6 weeks of age. There was no change in body weight when Egr-1 was blocked (Figure 5A). The efficiency of Egr-1 siRNA was ideal (Figure 5A). Interestingly, we found that the serum glucagon level of $d b$ / $d b$ mice was higher than that of control littermates, which were developing diabetes syndrome (Figure 5B), whereas the insulin sensitivity of these mice was normal at 4 weeks of age (data not shown). This result indicated that the abnormal hepatic gluconeogenesis, which results from the higher level of serum glucagon, might occur earlier than the insulin resistance during the development of type 2 diabetes. Notably, the fasting blood glucose level was reduced when the hepatic Egr-1 was blocked (Figure 5C). In this case, to investigate whether decreasing gluconeogenesis by knockdown of hepatic Egr-1 is beneficial for the improvement of insulin sensitivity, we then performed the PTT, GTT, and ITT at 4, 5, and 6 weeks of age, respectively. At 4 weeks, the PTT showed a slightly decreased hepatic gluconeogenesis, although the difference was not significant (Figure 5D). However, hepatic gluconeogenesis was largely impaired at 5 and 6 weeks of age after blocking hepatic Egr-1 (Figure 5, E and F). Sequentially, the glucose tolerance was improved slightly at 4 weeks of age and significantly at 5 and 6 weeks (Figure 5, G-I). Similarly, the insulin sensitivity was improved at 6 weeks of age, although a significant difference was not detected at 4 and 5 weeks of age (Figure 5, J-L). These results indicated that the abnormally increased hepatic gluconeogenesis that resulted from the high levels of serum glucagon played a role in the formation of insulin resistance during the development of type 2 diabetes and that Egr-1 acted as a key transcription factor in this progression.

\section{Discussion}

The sequential secretion of glucagon and insulin during fasting or postprandial states is critical to the maintenance of a constant level of blood glucose. Increased blood glucose after a meal will stimulate the secretion of insulin, which accelerates glucose uptake to cause a decrease in the blood glucose level. The lower glucose level during fasting stimulates the secretion of glucagon, which enhances gluconeogenesis to restore the blood glucose level. Thus, any type of inactivation of the insulin response or overactivity of glucagon might result in abnormal blood glucose levels and contribute to hyperglycemia in type 2 diabetes patients. Herein, we found that glucagon could stimulate Egr-1 activation, which was responsible for the expression of hepatic gluconeogenic genes during fasting stress. Through binding to the promoter of $\mathrm{C} / \mathrm{EBPa}$, which is a gluconeogenic transcription factor, Egr-1 indirectly activated the expression of gluconeogenic genes and facilitated glucagon function to maintain blood glucose level.

Glucagon has long been underestimated in the pathogenesis of type 2 diabetes, whereas insulin resistance and hyperinsulinism have traditionally been used to explain the formation of type 2 diabetes. In recent years, instead of the insulincentric theory, the glucagoncentric theory has been accepted as a better understanding of type 2 diabetes mellitus. ${ }^{5,14}$ As a hormone that regulates blood glucose levels, glucagon is most effective in promoting hepatic gluconeogenesis, which contributes to the abnormally increased endogenous glucose production in type 2 diabetes. ${ }^{6}$ Hyperglucagonemia is often seen in type 2 diabetes patients. ${ }^{32}$ In fact, even before any diabetic syndrome developed, 4-week-old $d b / d b$ mice already exhibited higher levels of serum glucagon (Figure 5B). Through knockdown of hepatic Egr-1 in $d b / d b$ mice, we found that insulin resistance could be ameliorated by reducing hepatic gluconeogenesis only at early stages before diabetic syndrome developed (Figure 5, D-L). Although we still do not fully understand the mechanism, considering the situation of the Egr-1/C/EBPa pathway in 8-week-old $d b / d b$ mice, we hypothesized that constitutive activation of Egr-1/C/EBPa is the key intracellular signaling mechanism by which hyperglucagonemia enhances hepatic gluconeogenesis. This finding that it is hyperglucagonemia that induces type 2 diabetes provides new evidence for the glucagoncentric theory.

As we know, cAMP/protein kinase A signaling is the canonical pathway for hepatic glucose production. ${ }^{33}$ Under fasting conditions, glucagon quickly induces increased hepatic glucose production through activation of cAMP/protein kinase A as soon as the blood glucose level begins to decrease. Glycogenolysis is the first step to produce glucose by the breakdown of liver glycogen. Because of the limited storage of liver glycogen, glycogenolysis only lasts for a short time and is followed by gluconeogenesis, which is essential for maintaining the blood glucose level after being fasted for a longer time. The cAMP/protein kinase A pathway mediates both glycogenolysis and gluconeogenesis. Herein, we propose that Egr-1/C/EBPa pathway also partially mediates the hepatic gluconeogenesis under glucagon stimulation and fasting condition. Notably, Egr-1 did not enhance the expression of gluconeogenic genes until 4 hours after glucagon stimulation (Figure 2, E-G), which suggests that Egr-1 was responsible for inducing the late-phase expression of gluconeogenic genes in response to glucagon. Thus, the Egr-1/C/EBPa signaling pathway may exert its role during the long-term fasting state to facilitate an increase in the blood glucose level by enhancing hepatic gluconeogenesis. Egr-1/C/EBPa could also be activated by glucagon and directly enhance the expression of gluconeogenic genes after glycogenolysis to maintain blood glucose homeostasis during long-term fasting condition.

Interestingly, we found that Egr-1 might exert different effects on cellular glucose metabolism on different stimuli in different tissues. In adipocytes, Egr-1 could inhibit the insulin signaling by hyperinsulinism, whereas in hepatocytes, it 
could enhance the gluconeogenesis by hyperglucagonemia. ${ }^{28}$ It has also been reported that the agonist of $5^{\prime}$-adenosine monophosphate-activated protein kinase reduces gluconeogenesis by inducing Egr-1 expression in H4IIE cells. ${ }^{17}$ In pancreas, glucose induces Egr-1 expression ${ }^{34}$; however, the proliferation of $\beta$ cells is enhanced by Egr-1, independent of glucose, and reduced Egr-1 expression may contribute to the beta cell failure in type 2 diabetes. ${ }^{35}$ Egr- 1 was also reported to mediate the insulin-induced smooth muscle cell proliferation and repair after injury. ${ }^{36}$ This could contribute to the pathophysiological characteristics of wound healing in diabetes. ${ }^{37}$ Therefore, the roles of Egr-1 in the cellular glucose metabolism are highly dependent on different stimuli. In addition, as is a transcription factor, Egr-1 is prone to regulate the effect of long-term cellular stimuli, such as hyperinsulinism or hyperglucagonemia.

Egr-1/C/EBPa is not the only pathway by which Egr-1 regulates hepatic glucose metabolism. As is reported by our previous study, Egr-1 exerts an important role in cellular insulin sensitivity by changing the balance between mitogenactivated protein kinase and phosphatidylinositol 3-kinase/ AKT signaling. ${ }^{28}$ This may also contribute to the hepatocytes' insulin sensitivity. Also, in this study, PGC-1a was found to be activated by Egr-1 in glucagon stimulation, although no direct binding site was found in the promoter of PGC-1a. Considering that PGC-1a is a pivotal component for accelerating cellular oxidation and hepatic gluconeogenesis, ${ }^{38}$ it is of interest to further explore the role of Egr-1 in hepatocyte energy expenditure and glucose metabolism.

Taken together, we found a new gluconeogenic pathway, Egr-1/C/EBPa, triggered by glucagon to maintain the blood glucose level under fasting conditions. Moreover, the constitutive activation of Egr-1/C/EBPa by hyperglucagonemia led to insulin resistance through abnormally increased hepatic gluconeogenesis. More important, we found that early intervention in hepatic Egr-1 expression might alleviate hyperglucagonemia-induced insulin resistance in $d b / d b$ mice (Figure 5). This finding will open a new avenue of research into the pharmaceutical treatment of type 2 diabetes mellitus.

\section{Acknowledgments}

Wild-type and dominant-negative early growth response gene-1 cDNAs were a gift from Prof. Jay M. Baraban (Johns Hopkins University, Baltimore, MD).

N.S. generated data and wrote the manuscript; S.J., X.Y., J.-M.L., S.-S.L., J.-Z.Z., and J.-L.Z. generated data; W.-W.T., X.-X.W., and N.X. contributed to discussion; B.X. reviewed/edited the manuscript; and C.-J.L. wrote and reviewed/edited the manuscript.

\section{References}

1. Saltiel AR, Kahn CR: Insulin signalling and the regulation of glucose and lipid metabolism. Nature 2001, 414:799-806
2. Weyer C, Funahashi T, Tanaka S, Hotta K, Matsuzawa Y, Pratley RE, Tataranni PA: Hypoadiponectinemia in obesity and type 2 diabetes: close association with insulin resistance and hyperinsulinemia. J Clin Endocrinol Metab 2001, 86:1930-1935

3. Kahn S: The relative contributions of insulin resistance and beta-cell dysfunction to the pathophysiology of type 2 diabetes. Diabetologia 2003, 46:3-19

4. Kahn SE, Hull RL, Utzschneider KM: Mechanisms linking obesity to insulin resistance and type 2 diabetes. Nature 2006, 444:840-846

5. Unger RH, Cherrington AD: Glucagonocentric restructuring of diabetes: a pathophysiologic and therapeutic makeover. J Clin Invest 2012, 122:4-12

6. Hundal RS, Krssak M, Dufour S, Laurent D, Lebon V, Chandramouli V, Inzucchi SE, Schumann WC, Petersen KF, Landau BR: Mechanism by which metformin reduces glucose production in type 2 diabetes. Diabetes 2000, 49:2063-2069

7. Natali A, Ferrannini E: Effects of metformin and thiazolidinediones on suppression of hepatic glucose production and stimulation of glucose uptake in type 2 diabetes: a systematic review. Diabetologia 2006, 49: 434-441

8. Ali S, Drucker DJ: Benefits and limitations of reducing glucagon action for the treatment of type 2 diabetes. Am J Physiol Endocrinol Metab 2009, 296:E415-E421

9. Dinneen S, Alzaid A, Turk D, Rizza R: Failure of glucagon suppression contributes to postprandial hyperglycaemia in IDDM. Diabetologia 1995, 38:337-343

10. Shah P, Basu A, Basu R, Rizza R: Impact of lack of suppression of glucagon on glucose tolerance in humans. Am J Physiol Endocrinol Metab 1999, 277:E283-E290

11. Shah P, Vella A, Basu A, Basu R, Schwenk WF, Rizza RA: Lack of suppression of glucagon contributes to postprandial hyperglycemia in subjects with type 2 diabetes mellitus. J Clin Endocrinol Metab 2000, 85:4053-4059

12. Hancock AS, Du A, Liu J, Miller M, May CL: Glucagon deficiency reduces hepatic glucose production and improves glucose tolerance in adult mice. Mol Endocrinol 2010, 24:1605-1614

13. Wang M-Y, Chen L, Clark GO, Lee Y, Stevens RD, Ilkayeva OR, Wenner BR, Bain JR, Charron MJ, Newgard CB: Leptin therapy in insulin-deficient type I diabetes. Proc Natl Acad Sci U S A 2010, 107: 4813-4819

14. Edgerton DS, Cherrington AD: Glucagon as a critical factor in the pathology of diabetes. Diabetes 2011, 60:377-380

15. Shen N, Yu X, Pan F-Y, Gao X, Xue B, Li C-J: An early response transcription factor, Egr-1, enhances insulin resistance in type 2 diabetes with chronic hyperinsulinism. J Biol Chem 2011, 286: $14508-14515$

16. Zhang J, Zhang Y, Sun T, Guo F, Huang S, Chandalia M, Abate N, Fan D, Xin H-B, Chen YE: Dietary obesity-induced Egr-1 in adipocytes facilitates energy storage via suppression of FOXC2. Sci Rep 2013, 3:1476

17. Berasi SP, Huard C, Li D, Shih HH, Sun Y, Zhong W, Paulsen JE, Brown EL, Gimeno RE, Martinez RV: Inhibition of gluconeogenesis through transcriptional activation of EGR1 and DUSP4 by AMPactivated kinase. J Biol Chem 2006, 281:27167-27177

18. Hamilton TB, Borel F, Romaniuk PJ: Comparison of the DNA binding characteristics of the related zinc finger proteins WT1 and EGR1. Biochemistry 1998, 37:2051-2058

19. Silverman ES, Collins T: Pathways of Egr-1-mediated gene transcription in vascular biology. Am J Pathol 1999, 154:665-670

20. Virolle T, Adamson ED, Baron V, Birle D, Mercola D, Mustelin T, de Belle I: The Egr-1 transcription factor directly activates PTEN during irradiation-induced signalling. Nat Cell Biol 2001, 3: 1124-1128

21. Yao J, Mackman N, Edgington TS, Fan ST: Lipopolysaccharide induction of the tumor necrosis factor-alpha promoter in human monocytic cells: regulation by Egr-1, c-Jun, and NF-kappa B transcription factors. J Biol Chem 1997, 272:17795 
22. Shen N, Shao Y, Lai S-S, Qiao L, Yang R-L, Xue B, Pan F-Y, Chen H-Q, Li C-J: GGPPS, a new EGR-1 target gene, reactivates ERK 1/2 signaling through increasing Ras prenylation. Am J Pathol 2011, 179:2740-2750

23. Chavrier P, Zerial M, Lemaire P, Almendral J, Bravo R, Charnay P: A gene encoding a protein with zinc fingers is activated during G0/G1 transition in cultured cells. EMBO J 1988, 7:29

24. Huang R-P, Liu C, Fan Y, Mercola D, Adamson ED: Egr-1 negatively regulates human tumor cell growth via the DNA-binding domain. Cancer Res 1995, 55:5054-5062

25. Hodge C, Liao J, Stofega M, Guan K, Carter-Su C, Schwartz J: Growth hormone stimulates phosphorylation and activation of elk-1 and expression of c-fos, egr-1, and junB through activation of extracellular signal-regulated kinases 1 and 2. J Biol Chem 1998, 273:31327-31336

26. Kang J-H, Kim M-J, Jang H-I, Koh K-H, Yum K-S, Rhie D-J, Yoon SH, Hahn SJ, Kim M-S, Jo Y-H: Proximal cyclic AMP response element is essential for exendin-4 induction of rat EGR-1 gene. Am J Physiol Endocrinol Metab 2007, 292:E215-E222

27. Keeton $\mathrm{AB}$, Bortoff $\mathrm{KD}$, Bennett WL, Franklin JL, Venable DY, Messina JL: Insulin-regulated expression of Egr-1 and Krox20: dependence on ERK1/2 and interaction with p38 and PI3-kinase pathways. Endocrinology 2003, 144:5402-5410

28. Yu X, Shen N, Zhang M-L, Pan F-Y, Wang C, Jia W-P, Liu C, Gao Q, Gao X, Xue B: Egr-1 decreases adipocyte insulin sensitivity by tilting PI3K/Akt and MAPK signal balance in mice. EMBO J 2011, 30: $3754-3765$

29. Wolfrum C, Shi S, Jayaprakash KN, Jayaraman M, Wang G, Pandey RK, Rajeev KG, Nakayama T, Charrise K, Ndungo EM: Mechanisms and optimization of in vivo delivery of lipophilic siRNAs. Nat Biotechnol 2007, 25:1149-1157
30. Morita M, Watanabe Y, Akaike T: Protective effect of hepatocyte growth factor on interferon-gamma-induced cytotoxicity in mouse hepatocytes. Hepatology 1995, 21:1585-1593

31. Emilsson V, Liu Y-L, Cawthorne MA, Morton NM, Davenport M: Expression of the functional leptin receptor mRNA in pancreatic islets and direct inhibitory action of leptin on insulin secretion. Diabetes 1997, 46:313-316

32. Fineman M, Weyer C, Maggs D, Strobel S, Kolterman O: The human amylin analog, pramlintide, reduces postprandial hyperglucagonemia in patients with type 2 diabetes mellitus. Horm Metab Res 2002, 34: 504-508

33. Koo S-H, Flechner L, Qi L, Zhang X, Screaton RA, Jeffries S, Hedrick S, $\mathrm{Xu}$ W, Boussouar F, Brindle P: The CREB coactivator TORC2 is a key regulator of fasting glucose metabolism. Nature 2005, 437:1109-1111

34. Josefsen K, Sørensen L, Buschard K, Birkenbach M: Glucose induces early growth response gene (Egr-1) expression in pancreatic beta cells. Diabetologia 1999, 42:195-203

35. Garnett KE, Chapman P, Chambers JA, Waddell ID, Boam DS: Differential gene expression between Zucker Fatty rats and Zucker Diabetic Fatty rats: a potential role for the immediate-early gene Egr-1 in regulation of beta cell proliferation. J Mol Endocrinol 2005, 35:13-25

36. Gousseva N, Kugathasan K, Chesterman CN, Khachigian LM: Early growth response factor-1 mediates insulin-inducible vascular endothelial cell proliferation and regrowth after injury. J Cell Biochem 2001, 81:523-534

37. Yechoor VK, Patti M-E, Saccone R, Kahn CR: Coordinated patterns of gene expression for substrate and energy metabolism in skeletal muscle of diabetic mice. Proc Natl Acad Sci U S A 2002, 99: 10587-10592

38. Handschin C, Spiegelman BM: The role of exercise and PGC1 $\alpha$ in inflammation and chronic disease. Nature 2008, 454:463-469 